\title{
APLICACIÓN DEL TRABAJO EN EQUIPO EN COLOMBIA, ADELANTOS INVESTIGATIVOS
}

\author{
Víctor Manuel Ardila Soto \\ Docente Universidad Francisco de Paula Santander \\ Grupo de Investigación Proyección Empresarial \\ vmardila@bari.ufps.edu.co
}

\section{RESUMEN}

El presente artículo, es resultado de una investigación empírica desarrollada sobre el concepto de trabajo en equipo, que tienen los directivos de las empresas de producción manufacturera en las ciudades de Bogotá, Cali, Medellín, Barranquilla, Bucaramanga y Cúcuta, con base en una conceptualización ajustada de acuerdo a el aporte realizado por diferentes autores (Mueller, Procter, Buchanan, 2000; Knights y McCabe, 2000; Sewell, 1999; Tranfield et al. 1999; Katzenbach y Smith, 1993 y Montebello 2000), divulgándose como resultado el caso colombiano en lo concerniente a este concepto, como aporte investigativo en ciencias sociales en lo relacionado a trabajo en equipo.

Palabras claves:

Trabajo en equipo

Concepto

Empresas colombianas

\section{INTRODUCCIÓN}

El presente artículo, se elabora con base en el trabajo de investigación titulado "Estudio de las empresas colombianas del sector de la producción manufacturera para determinar la aplicación del trabajo en equipo en ellas", el cual busca identificar las empresas que realmente aplican trabajo en equipo en Colombia, ubicándolas de acuerdo a la relación de interrogantes como el concepto y su relación con el cargo y la ciudad de ubicación de las empresas objeto del estudio.

El contenido sintético está relacionado con los resultados hasta ahora identificados en la investigación en lo referente al concepto que tienen los directivos de las empresas de producción manufacturera de cien o más empleados sobre el trabajo en equipo que se realiza en Colombia y una discusión sobre el tema, con el objeto de generar inquietud sobre la organización interna de las empresas en equipos de trabajo.

\section{ANTECEDENTES}

Es normal encontrar empresas que manifiesten que su forma de trabajo es en equipo, por ello surge la inquietud: ¿̇realmente las empresas que dicen trabajar en equipo, lo hacen? (Ardila, 2002). Para resolver este dilema se desarrolló la presente investigación, que toma como referencia las generalidades y antecedentes presentados en Ardila (2002, 2003), que son: Tranfield et al, (1999), en su artículo "Rediseñando las organizaciones para la mejora industrial"; Bacon y Blyton (2000), en su artículo "Profundidad y Superficialidad del trabajo en equipo: Percepciones de la manera de pensar de la dirección y efectos sobre los recursos humanos y la organización"; Mueller, Procter y Buchanan (2000), presentan su artículo "el trabajo en equipo en su contexto: Los antecedentes, naturaleza y dimensiones"; Delbridge, Lowe y Oliver (2000), en su artículo "Responsabilidades del trabajo en equipo en los niveles más bajos de la organización"; Knights y McCabe (2000), en su artículo "Embrujados, molestos y confundidos: el significado y experiencia de trabajar en equipo para los empleados de una empresa automotriz"; Sprigg, Jackson y Parker (2000); "Producción trabajo en equipo: La importancia de la interdependencia y la autonomía para la tensión y satisfacción del empleado"; finalmente, Geary y Dobbins (2001), a través de su artículo "trabajo en equipo: Otra manera de conseguir el control, por parte de la dirección".

\section{PROBLEMA}

En lo referente a la aplicación del trabajo en equipo se presentan dos tipos de resultados; las empresas que han tenido éxito con su aplicación (Mueller, Procter y Buchanan, 2000; Sprigg, Jackson y Parker, 2000) y 
Aplicación del trabajo en equipo en Colombia, adelantos investigativos

Víctor Manuel Ardila Soto

las empresas que han fracasado con su aplicación (Lawler, 1986, 1988; Saporito, 1986; Verespej, 1990; Walton, 1985; Sprigg, Jackson y Parker, 2000). Esta diferencia en los resultados generó la inquietud de analizar que pasa en Colombia en lo referente a este tema, que inicia con la necesidad de conocer el concepto que tienen los directivos de las empresas de la producción manufacturera que dicen que aplican trabajo en equipo actualmente en Colombia, y corroborarlo con un concepto definido para determinar si realmente dichas empresas aplican el trabajo en equipo o simplemente creen estar haciéndolo. De esta manera se proyecta analizar que pasa en Colombia en lo referente a trabajo en equipo desde la óptica de los directivos con relación al concepto real.

\section{MARCO CONCEPTUAL}

Se podría defender que el concepto de trabajo en equipo en las sociedades industrializadas occidentales tiene connotaciones de colaboración, jovialidad, camaradería, y compromiso en el trabajo (Mueller, Procter, Buchanan, 2000). Sin embargo, analizando algunos artículos como los de Tranfield et al. (1999), Knights y McCabe (2000) y el mismo Mueller, Procter y Buchanan (2000), no es conveniente emitir un concepto de manera rápida y menos en países como Colombia, para lo que se requiere profundizar en el tema y, sobretodo, en la conceptualización que presentan los diferentes autores en busca de unificar criterios y facilitar la orientación de la presente investigación en lo referente a concepto y aceptación.

El trabajo en equipo está definido en el diccionario como dos o más personas que trabajan para lograr una meta común o compartida en virtud de su colaboración (Sewell, 1999); esta definición es genérica y no distingue variables claves en el desarrollo del trabajo en equipo.

Un equipo es un grupo de empleados, normalmente entre tres y quince miembros que se encuentran con un poco de regularidad para trabajar interdependientemente en cumplir una tarea específica (Mueller, Procter y Buchanan, 2000); esta definición integra una nueva variable "la interdependencia".

Tranfield et al. (1999), reúne varios conceptos entre los cuales está el de Katzenbach y Smith (1993), asesores de McKinsey, definición enunciada también por Montebello (2000), que define un equipo como: "un número reducido de personas con habilidades complementarias, que están comprometidas con un propósito común, un método y unas metas de desempeño, por los cuales se responsabilizan mutuamente". Los elementos importantes de esta definición son las habilidades complementarias, el propósito común y la responsabilidad mutua; sin embargo a medida que se profundiza en el artículo se clarifica más este concepto donde manifiesta que el trabajo en equipo comprende una forma de colectividad que tiene las características de demostrar un propósito compartido y se desarrolla a través de una serie de roles interdependientes; es decir, unifica el concepto inicial y lo correlaciona con el de Mueller, Procter y Buchanan (2000) al integrar propósito e interdependencia. Finalmente Tranfield et al. (1999), complementa su concepto identificando el trabajo en equipo como una intervención de mejora de actuación estratégica con que las compañías relacionan la reingeniería de la organización para desarrollar una ventaja competitiva. Al analizar esta definición se encuentran aspectos interrelacionados importantes como son: grupo de personas o colectividad, habilidades, compromiso, meta, propósito común, papeles interdependientes, mejora de la actuación y finalmente ventaja competitiva.

Knights y McCabe (2000) utilizan un concepto más especializado de acuerdo con el estudio del caso en la compañía automovilística del reino Unido y dicen: "El trabajo en equipo es involucrar en el equipo, personal de producción orientado por los líderes de equipo. Dentro de cada equipo los miembros serán totalmente flexibles y responsables de todas las actividades que tienen lugar en ese departamento" 
Aplicación del trabajo en equipo en Colombia, adelantos investigativos

Víctor Manuel Ardila Soto

este concepto incluye otras variables como líder de equipo, flexibilidad y responsabilidad.

En definitiva el concepto de Katzenbach y Smith (1993) es el más claro y está sustentado por autores como Tranfield et al., (1999); Montebello (2000), por lo cual se tomó como el más ajustado en la aplicación de la encuesta Ardila (2004).

\section{POBLACION Y MUESTRA}

El tipo de estudio a aplicar en esta etapa del proceso es exploratorio, el cual se requiere ante la necesidad de ubicar información, de un tema o problema de investigación que realmente ha sido poco estudiado en Colombia de manera empírica o a través de trabajo de campo, es decir, que revisada la literatura, reveló que únicamente hay guías relacionadas con el problema de estudio, situación planteada por Ardila (2003); por lo cual, se toma como fuente de información, la fuente primaria que permite recoger de primera mano de las empresas de producción manufacturera de cien o más empleados ubicadas en las ciudades de Bogotá, Cali, Medellín, Barranquilla, Bucaramanga y Cúcuta, para lo cual se procedió a solicitar las bases de datos a las cámaras de comercio de las ciudades respectivas, definiéndose la población total como: Bogotá (340), Medellín (89), Cali (107), Barranquilla (43), Bucaramanga (16) y Cúcuta (6), para una población total de 601 empresas.

Igualmente se utiliza los datos cuantitativos, utilizando la encuesta como método. Este método de recopilación de información cuenta con preguntas estructuradas (estandarizadas); es decir, que se utiliza un cuestionario formal que presenta las preguntas en un orden predeterminado y se aplicó enviándolo por correo electrónico y por fax.

Tamaño de la Muestra: para el tamaño de la muestra se tuvo en cuenta una probabilidad inicial de respuesta correcta $p=0.956$ y una probabilidad inicial de respuesta incorrecta $q=1-p=0.044$, un nivel de confianza del 1.96, y un margen de error de $4 \%$. Con esta información se aplicó las siguientes formulas:

$$
\begin{aligned}
& \mathrm{n}_{0}=\frac{\mathrm{p} \cdot \mathrm{q} \cdot \mathrm{z}^{2}}{\mathrm{e}^{2}} \\
& \mathrm{n}=\frac{\mathrm{n}_{0} \cdot \mathrm{N}}{\mathrm{n}_{0}+(\mathrm{N}-1)} \\
& \mathrm{n}_{0}=\frac{0.956 * 0.044^{*}(1.96)^{2}}{(0.04)^{2}}
\end{aligned}
$$

$\mathrm{n}_{0}=101$ Tamaño de muestra para poblaciones infinitas.

$\mathrm{n}=\frac{101 * 591}{101+(591-1)}$

$\mathrm{n}=86$ tamaño que se extiende a 90 para redondear fracciones de encuestas

De las cuales su distribución se hizo de la manera siguiente:

Tabla 1. Distribución del Tamaño de la Muestra (n)

\begin{tabular}{|l|c|c|c|c|}
\hline \multicolumn{1}{|c|}{ CIUDAD } & $\begin{array}{c}\text { No. de } \\
\text { empresas } \mathrm{N}\end{array}$ & $\% \mathrm{~N}$ & $\mathrm{n}$ esperada & $\mathrm{n}$ Real \\
\hline Bogotá & 330 & $55.84 \%$ & 50 & 44 \\
\hline Medellín & 89 & $15.06 \%$ & 14 & 16 \\
\hline Cali & 107 & $18.10 \%$ & 16 & 14 \\
\hline Barranquilla & 43 & $7.28 \%$ & 7 & 7 \\
\hline Bucaramanga & 16 & $2.71 \%$ & 2 & 3 \\
\hline Cúcuta & 6 & $1.02 \%$ & 1 & 6 \\
\hline TOTAL & 591 & $100 \%$ & 90 & 90 \\
\hline
\end{tabular}

Como técnica de muestreo, se tomó el muestreo de grupos, donde, la población meta se divide primero en subpoblaciones o grupos, recíprocamente selectas, llamadas grupos y después se seleccionan una muestra aleatoria de grupos con base en una técnica de muestreo probabilística. Para cada grupo 
Aplicación del trabajo en equipo en Colombia, adelantos investigativos

Víctor Manuel Ardila Soto

seleccionado, se incluyen todos los elementos en la muestra o se toma una muestra de elementos en forma probabilística.

El porcentaje de respuesta de la muestra fue alto, pues el $98.9 \%$ respondió la pregunta relacionada con el concepto de trabajo en equipo.

\subsection{RESULTADOS PARCIALES DE LA INVESTIGACIÓN}

Pregunta: Cargo de la persona que diligencia la encuesta

La distribución de los cargos de las personas encuestadas es:

Tabla 2. Cargo de los Entrevistados

\begin{tabular}{|l|c|c|}
\hline \multicolumn{1}{|c|}{ CARGO } & Total & Total \\
\hline Asesor & 1 & 1,1 \\
\hline Asistente & 6 & 6,7 \\
\hline Director Área & 28 & 31,1 \\
\hline Director RH & 23 & 25,6 \\
\hline Gerente & 25 & 27,8 \\
\hline N.R. & 1 & 1,1 \\
\hline Presidente & 3 & 3,3 \\
\hline Sub-gerente & 3 & 3,3 \\
\hline Total & 90 & 100,0 \\
\hline
\end{tabular}

Para facilitar la interpretación, se presenta el presente gráfico:

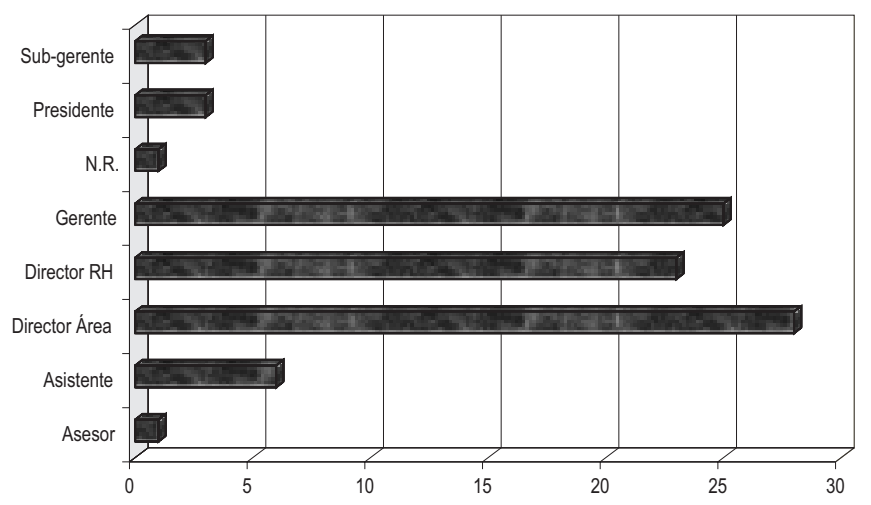

Gráfico 1. Cargo de Encuestados
Quienes más participaron en el diligenciamiento de la encuesta, fueron los gerentes de las empresas, los directores de recursos humanos y directores de otras dependencias (84.5\% en total), mostrando la seriedad y responsabilidad con que acogieron la investigación, lo que garantiza la calidad de la misma.

Pregunta: Para usted trabajo en equipo es:

Se presentaron cinco posibles respuestas, de acuerdo con la conceptualización definida anteriormente. De estos conceptos, solo uno es el verdadero, el presentado por Montebello (2000). Sin embargo, los encuestados al diligenciar el formulario, seleccionaron en ocasiones algunas combinaciones, por ello es importante aclarar el significado de las variadas respuestas relacionadas en la tabulación de la pregunta. Estas son:

\section{1, 2, 4, Err = Concepto errado}

Real $=$ Concepto verdadero 0 real

T/Ant = Todas las anteriores, es decir seleccionan la respuesta correcta y las erradas $(1,2,4)$

Comb = seleccionan la respuesta correcta y la combinan con conceptos errados $(1,2,4)$.

Err $=$ Seleccionan un concepto errado 0 combinaciones de ellos $(1,2,4)$

NR $\quad=$ No responde.

Con base en lo anterior, el resultado obtenido es:

Tabla 3. Resultados de la Encuesta

\begin{tabular}{|c|c|c|}
\hline CONCEPTO & TOTAL & $\%$ \\
\hline $1,2,4$, Err. & 33 & 36.6 \\
\hline Real & 22 & 24,4 \\
\hline T/Ant & 25 & 27,8 \\
\hline Comb & 9 & 10,0 \\
\hline NR & 1 & 1,1 \\
\hline Total & 90 & 100,0 \\
\hline
\end{tabular}


Aplicación del trabajo en equipo en Colombia, adelantos investigativos

Víctor Manuel Ardila Soto

Sólo un $24.4 \%$ de los encuestados tienen un concepto real y claro de qué es trabajo en equipo; un $36.6 \%$ definen trabajo en equipo con conceptos no acertados $(1,2,4)$; un $37.8 \%$ incluye la respuesta real con otro tipo de respuestas (T/Ant, Comb), es decir no están muy claros en el concepto; finalmente, un $1.1 \%$ no respondió la pregunta.

\subsection{DISCUSIÓN}

Al observar un nivel no significativo de conocimiento del concepto de trabajo en equipo, genera la necesidad de profundizar en la interpretación de esta tabla combinándola con otras variables como: primero, el cargo de las personas encuestadas; segundo, con la ciudad; de lo cual, se obtiene la tabla 4.

Los gerentes y directores de área expresaron un concepto más acertado de trabajo en equipo $(7.8 \%$ $\mathrm{c} / \mathrm{u})$; contrario a esto fueron pocos los directores del área de recursos humanos que acertaron con el concepto (3.3\%).

Al analizar los conceptos errados (1,2,4, Err), los directores de área y los directores de recursos humanos son quienes presentan un concepto errado en mayor proporción $(12.2 \% \mathrm{c} / \mathrm{U})$, con relación a los gerentes (6.6\%).

Quienes combinan el concepto real con otros conceptos (Comb, T/Ant) en mayor proporción son los gerentes (12.3\%).

Relación concepto/ciudad: al combinar el concepto que tienen los directivos, con la ciudad, se obtiene la tabla 5 .

Entre los directivos que respondieron acertadamente (Verd), con relación a la ciudad, se observa que en Bucaramanga y Bogotá $133.3 \%$ y $29.5 \%$ respectivamente) fueron las ciudades de mejor concepto. Por el contrario, las ciudades donde se respondió menos con el concepto real son; Cúcuta y Barranquilla (14.3\% y $16.7 \%$, respectivamente).

Las ciudades, que presentan un concepto errado en mayor escala son Cúcuta y Cali (50\% c/u).

La ciudad que más seleccionó combinaciones de respuestas, que incluye trabajo en equipo, es Barranquilla (71.4\%), esto los caracteriza como los indecisos.

Tabla 4. Relación concepto cargo

\begin{tabular}{|c|c|c|c|c|c|c|c|c|c|}
\hline \multirow{2}{*}{ CONCEPTO } & \multicolumn{9}{|c|}{ CARGO } \\
\hline & Asesor & Asistente & Director Área & Director RH & Gerente & N.R. & Presidente & Sub-Gerente & Total \\
\hline 1 & & & 2,2 & & 1,1 & & & & 3,3 \\
\hline 2 & & & 1,1 & 4,4 & 1,1 & & & & 6,7 \\
\hline Real & & 1,1 & 7,8 & 3,3 & 7,8 & & 3,3 & 1,1 & 24,4 \\
\hline 4 & 1,1 & 1,1 & 7,8 & 6,7 & 4,4 & 1,1 & & 2,2 & 24,4 \\
\hline T/Ant & & 3,3 & 10,0 & 7,8 & 6,7 & & & & 27,8 \\
\hline Comb & & 1,1 & 1,1 & 2,2 & 5,6 & & & & 10,0 \\
\hline Err & & & 1,1 & 1,1 & & & & & 2,2 \\
\hline NR & & & & & 1,1 & & & & 1,1 \\
\hline Total & 1,1 & 6,7 & 31,1 & 25,6 & 27,8 & 1,1 & 3,3 & 3,3 & 100,0 \\
\hline
\end{tabular}


Aplicación del trabajo en equipo en Colombia, adelantos investigativos

Víctor Manuel Ardila Soto

Tabla 5. Relación concepto ciudad

\begin{tabular}{|c|c|c|c|c|c|c|c|}
\hline \multirow{2}{*}{ CONCEPTO } & \multicolumn{7}{|c|}{ CIUDAD } \\
\cline { 2 - 8 } & Bucaramanga & Barranquilla & Bogotá & Cali & Cúcuta & Medellín & Total \\
\hline 1 & & & 4,5 & & & 6,3 & 3,3 \\
\hline 2 & & 14,3 & 2,3 & 14,3 & & 12,5 & 6,7 \\
\hline Verd & 33,3 & 14,3 & 29,5 & 21,4 & 16,7 & 18,8 & 24,4 \\
\hline 4 & 33,3 & & 27,3 & 28,6 & 50,0 & 12,5 & 24,4 \\
\hline T/Ant & 33,3 & 57,1 & 22,7 & 21,4 & 33,3 & 31,3 & 27,8 \\
\hline Comb & & 14,3 & 11,4 & 7,1 & & 12,5 & 10,0 \\
\hline Err & & & & 7,1 & & 6,3 & 2,2 \\
\hline NR & & & 2,3 & & & & 1,1 \\
\hline Total & 100,0 & 100,0 & 100,0 & 100,0 & 100,0 & 100,0 & 100,0 \\
\hline
\end{tabular}

\section{CONCLUSIONES}

La encuesta la diligenciaron en su totalidad directivos de la empresa, tales como: gerentes, directores de áreas diferentes a recursos humanos, recursos humanos, presidentes de empresas, subgerentes y asesores de empresas, situación que se identifica con la muestra determinada.

Entre los grupos de directivos que se tuvieron en cuenta, se observó que: quienes más se ajustan al concepto de Katzenbach y Smith (1993) son los directores de área y los gerentes, sin embargo, es importante tener en cuenta que los pocos presidentes de empresas que diligenciaron la encuesta seleccionaron esta respuesta como única opción. Los directores de recursos humanos son quienes tienen en menor proporción un concepto acertado de trabajo en equipo, igualmente son las personas que tienen otros conceptos de trabajo en equipo. Para lo cual, si se desea aplicar trabajo en equipo desde la óptica del concepto presentado en la conceptualización, deben ser preparados y capacitados en mayor proporción con relación a los directores de otras áreas o a los gerentes.

Los directivos en Colombia en un alto porcentaje piensan que están aplicando innovación administrativa en lo referente a trabajo en equipo y posiblemente no lo estén haciendo. Es decir, no existe un concepto claro de trabajo en equipo, pues solo un $24.4 \%$ ha demostrado a través de la respuesta a una pregunta de selección múltiple conocer el concepto de Katzenbach y Smith (1993) seleccionado como el real. Lo que genera inquietud y preocupación, al pensar en la posibilidad de que estos conceptos, enfoques y teorías actuales se tomen a la ligera con base en un conocimiento limitado resultado de alguna lectura del tema, lo cual puede ocasionar más complicaciones que beneficios ante la necesidad de improvisación.

Finalmente, es importante resaltar que está en revisión el resultado relacionado con otras variables como ciudad, y los conceptos de número de equipos emitidos por autores como Jackson y Parker; (2000), Mueller, Procter y Buchanan (2000); Delbridge, Lowe y Oliver (2000). Así mismo, es conveniente darle continuidad a la presente investigación a través de un tercer estudio que tiene como objetivo general evaluar la eficacia en las empresas del sector de la producción manufacturero de cien o más empleados que aplican trabajo en equipo y que se identifican con el concepto real, expuesto por Katzenbach y Smith (1993) y las empresas que se identifican con otros conceptos. De esta manera se podrá brindar una información más 
Aplicación del trabajo en equipo en Colombia, adelantos investigativos

Víctor Manuel Ardila Soto

completa a los gerentes en Colombia y a los investigadores nacionales e internacionales interesados en el caso colombiano en lo referente a este concepto "trabajo en equipo".

\section{BIBLIOGRAFÍA}

ARDILA, Víctor M., Teamworking, conceptualización, aplicación y aceptación por parte de los directivos y empleados en las empresas de producción; artículo publicado en el libro: Cambio Organizacional en el contexto de la cultura latinoamericana, ASCOLFA, 2002.

ARDILA, Víctor M., Trabajo en Equipo, conceptualización y aceptación por parte de directivos y empleados en las empresas de producción manufacturera, investigación suficiencia investigadora, Universidad Pablo de Olavide, Sevilla, España, 2003.

BACON, Nicolas \& BLYTON, Paul. High road and low road Teamworking: Perceptions of management rationales and organizational and human resource outcomes.; Human Relations, Nov 2000 v53 i1 1. $1425 \mathrm{p}$.

DELBRIDGE, Rick; LOWE, James \& OLIVER, Nick; Shopfloor responsibilities under lean Teamworking; Human Relations, 2000. 1459 p.

GEARY, John F.; DOBBINS Anthony. Teamworking: A new dynamic in the pursuit of management control; Human Resource Management Journal, London; 2001 ; Vol. 11, iss. 1. p. 3, 21.

KATZENBACH J. R. y SMITH D.K., The Wisdomof Teams, Boston, H.B.S. Press, 1993.

KNIGHS, David \& MCCABE, Darren. Bewitched, bothered and bewildered: The meaning and experience of Teamworking for employees in an automobile company. Human Relations, Nov 2000 v53 i1 1. 1481 p.

LAWLER, E.E. High involvement management: Participative strategies for improving organizational performance. San Francisco, CA: Jossey Bass, 1986.

MONTEBELLO, Antony R., Equipos de trabajo extraordinarios: habilidades para dirigir una organización. Editorial Pax México, 2000.

MUELLER, F., PROCTER Stephen \& BUCHANAN David. Teamworking in its context(s): Antecedents, nature and dimensions. Human Relations, Nov 2000. 1358 p.

SAPORITO, B. The revolt against 'working smarter'. Fortune, 1986, 114. p. 58 - 65.

SEWELL, G. The discipline of teams: The control of team-based industrial work through electronic and peer surveillance. Administrative Science Quarterly, 1999, 43(2). p. 397 - 428.

SPRIGG, Christine A; JACKSON, Paul R y PARKER, Parker. Production Teamworking: The importance of interdependence and autonomy employee strain and satisfaction. Human relations, Nov 2000, v53 i1 1. $1519 \mathrm{p}$.

TRANFIELD, David et al. Teamworking: Redesigning the organization for manufacturing improvements; Technology Analysis y Strategic Management, Abingdon, jun 1999; vol. 11, iss. 2. 50 p.

VERESPEJ, J.A. When you put a team in charge. Industry Week, 1990. p. 239, 30 - 2.

WALTON, R.E. From control to commitment in the workplace. Harvard Business Review, 1985. p. 63, $77-84$. 